\section{Early intervention and recovery for young people with early psychosis: consensus statement ${ }^{1 *}$}

\author{
J. BERTOLOTE and P. McGORRY \\ on behalf of the World Health Organization \\ and the International Early Psychosis Association
}

Yet, despite the availability of interventions that can reduce relapses by more than $50 \%$, not all affected individuals have access to them, and when they do, it is not always in a timely and sustained way. Among the goals of care to these people, the identification of the illness and its treatment, as early as possible, represents a high priority.

Comprehensive programmes for the detection and treatment of early psychosis and in supporting the needs of young people with early psychosis carry the important function of promoting recovery, independence, equity and self-sufficiency and of facilitating the uptake of social, educational and employment opportunities. These programmes can be provided by individuals and teams with specialised skills, with a full range of primary healthcare services for every young person with early psychosis.

Prompt and effective interventions for young people with early psychosis, for their families, close friends and other carers of areas of medicine. This document attempts this task for early intervention in psychotic disorders. It was originally inspired by the St Vincents declaration on the care of diabetes and carefully developed by David Shiers and Jo Smith with support from the Initiative to Reduce the Impact of Schizophrenia, National Institute for Mental Health in England and Rethink, resulting in the UK-focused Newcastle Declaration. The World Health Organization and the International Early Psychosis Association then collaborated to produce an international version of the declaration, which articulates the universal principles of early intervention and tries to blend these with local capacities and cultural diversity.

According to the World Health Organization's World Health Report 2001 (World Health Organization, 2002), schizophrenia and other forms of psychoses that affect young people represent a major public health problem. Worldwide, they rank as the third most disabling condition (following quadriplegia and dementia and higher than blindness and paraplegia) and pose an enormous burden, both in terms of economic cost and of human suffering.

I. This document is based on the UK Newcastle Declaration (2002), an idea conceived by IRIS, the Initiative to Reduce the Impact of Schizophrenia, and further developed with the support of Rethink and the National Institute for Mental Health in England. * Paper presented at the Third International Early Psychosis Conference, Copenhagen, Denmark, September 2002.

Table I Five-year outcomes that a comprehensive and effective programme would deliver to people with early psychosis and their families

Comprehensive programme
Improving access and engagement
Walk-in responsive services usually
provided in primary care settings should
be equipped to deal effectively with early
psychosis.
Service interfaces are designed to support
quicker and more effective engagements
of young people.

Raising community awareness

About the importance and the opportunities for earlier detection and improved management of psychosis.

\section{Promoting recovery}

Services should enable rather than disable, ultimately aspiring to healing and recovery.

This means receiving encouragement and sufficient support to retain/regain full participation in society.

\section{Family engagement and support} Families have better access to information and education, social, economic, practical and emotional support.

\section{Practitioners training}

All primary care sites are equipped to deal effectively with early psychosis. Continued professional development is supported for all specialist staff working with young people with psychosis.
Measured outcome

The mean duration of untreated psychosis from the onset of psychosis is less than 3 months.

The use of involuntary treatments in the first engagement is less than $25 \%$.

Suicide rates within the first 2 years from diagnosis will be less than I\%.

All I5-year-olds are equipped by mainstream education to understand and deal with psychosis.

Psychosis-specific training is available to teachers and other relevant community agencies.

2 years after diagnosis $90 \%$ of affected individuals have employment/education rates similar to their age-/ gender-matched peers.

2 years after diagnosis $90 \%$ of affected individuals will report satisfaction with their employment, educational and social attainments.

Days out of role over 2 years post diagnosis will be no more than $25 \%$

Services will give a meaningful response to families or key supporters within I week.

$90 \%$ of families will feel respected and valued as partners in care.

Recognition, care and treatment of young people with psychosis is a routine part of training curricula of all primary care and social care practitioners. Specific early intervention training programmes are resourced and evaluated. 
Table 2 Recommended minimum 5 -year strategic actions required for the care of people with early psychosis

\begin{tabular}{lll}
\hline $\begin{array}{l}\text { Ten overall strategic } \\
\text { recommendations }\end{array}$ & $\begin{array}{l}\text { Scenario A } \\
\text { Low level of resources }\end{array}$ & $\begin{array}{l}\text { Scenario B } \\
\text { Medium level of resources }\end{array}$ \\
\hline $\begin{array}{l}\text { I. Provide treatment } \\
\text { in primary care: early }\end{array}$ & $\begin{array}{l}\text { Primary care health personnel } \\
\text { can recognise and initiate treat- } \\
\text { to comprehensive community- }\end{array}$ & $\begin{array}{l}\text { Pilot care pathway audits } \\
\text { of those developing early } \\
\text { psychosis to improve detection }\end{array}$ \\
\hline
\end{tabular}

Scenario C

High level of resources

based mental health services.

2. Make psychotropic and psychosocial interventions available

\section{Provide care}

in the community: move towards least restrictive and least coercive service approaches.

\section{Educate the public: raise community awareness about the importance and the opportunities for earlier detection and improved management of psychosis.}

5. Involve communities, families and consumers
Low-dose typical antipsychotic and other relevant medications are available and offered routinely to people with early psychosis in all healthcare settings.

Practical psychosocial support available via family, friends and community organisers.

Move away from institutional and custodial models of care for those with early psychosis who have a persistent and disabling course and provide community care wherever possible for early psychosis.

Preferred in-patient facility of a general hospital psychiatric unit rather than traditional mental hospital.

Support public campaigns to raise awareness about mental illness in young people. Support non-government organisations in public education.

Support the formation of self-help groups for young people with early psychosis, family members and key supporters. Fund schemes for non-governmental organisations and mental health initiatives.
Initiate pilot mental health programmes to provide comprehensive care and treatment, i.e. psychotropic and psychosocial: the latter available to consumer and family. Low-dose atypical antipsychotic medication is available and offered routinely in all healthcare settings.

Initiate schemes which divert people with early psychosis presenting to criminal justice services, more appropriately into mental health services.

Use of involuntary treatment in early psychosis is measured routinely.

Support campaigns directed at young people which reduce stigma and discrimination against As part of mainstream education all young mental illness.

Initiate evidence-based mental health promotion and early intervention programmes in collaboration with other sectors.

Pilot initiatives that help families and key supporters access help and information about early psychosis.

Representation of communities, consumers and families in services and policy-making.
Strategies should be developed for reducing the time taken to access support from the onset of psychosis (duration of untreated psychosis).

Integrated care pathways are routinely evaluated based on agreed referral guidelines between primary care, relevant community agencies, and specialist services. Special access channels to be developed.

Low-dose atypical antipsychotics are offered as medication of first choice.

Cognitive-behavioural therapy is routinely available.

Family interventions are routinely available. The following treatment programmes are routinely provided: vocational recovery; continuing care, relapse prevention; substance misuse harm reduction.

Development of strategies for minimising the use of involuntary treatments in early psychosis (e.g. home-based intervention, alternative residential settings).

'Streamed' youth-focused in-patient services are routinely available.

Develop youth-friendly day programmes.

Develop youth-oriented residential facilities to support independent living for young people with psychosis.

Strategies for reducing risk of suicide for people with early psychosis are developed. Individuals with early psychosis are offered treatment and care in community settings wherever possible.

Launch public campaigns to raise awareness about the recognition and treatment of early psychosis. people are taught about psychosis.

Psychosis awareness training is provided to teachers, youth and welfare workers, police and criminal justice services and other relevant community agencies.

Families and key supporters will receive effective services appropriate to their needs, and access to advocacy support as appropriate. Services should systematically demonstrate effectiveness in terms of:

(a) early engagement of families and close friends;

(b) whether families feel valued as partners in care. 
Table 2 (Continued)

\begin{tabular}{ll}
\hline $\begin{array}{l}\text { Ten overall strategic } \\
\text { recommendations }\end{array}$ & $\begin{array}{l}\text { Scenario A } \\
\text { Low level of resources }\end{array}$ \\
\hline $\begin{array}{l}\text { 6. Establish national policies, } \\
\text { programmes and legislation }\end{array}$ & $\begin{array}{l}\text { Formulate mental health pro- } \\
\text { grammes and policy. } \\
\text { Increase the budget for mental } \\
\text { healthcare to at least 5\% of gross } \\
\text { domestic product. }\end{array}$ \\
\end{tabular}

\begin{tabular}{ll}
\hline Scenario B & Scenario C \\
Medium level of resources & High level of resources
\end{tabular}

Mevelop policy and financial

commitment to improve services development, training and policy-making.

for people with early psychosis

and their families.

Growth in mental health

expenditure focused on young

people with early psychosis.

Early intervention in psychosis is embraced as a key national priority demonstrated by investment of resources.

Specific commitment in terms of policy and health spending which underpin services for young people with psychosis and their families.

7. Develop human resources

Primary healthcare practitioners Specialist mental health practirecognise, through awareness tioners receive specific training training, that young people with about the recognition, care and psychosis have their own legiti- treatment of young people with mate mental health concerns early psychosis. and needs.

Awareness training of psychiatrists and nurses about early psychosis care and treatment.

Specialist mental health practitioners develop advanced skills and knowledge about early detection, care and treatment of psychotic disorders in young people.

Recognition, care and treatment of young people with psychosis and their families should form a routine part of the training curricula of all primary (generalist) health and social care practitioners. Psychosis awareness training is provided to teachers, youth and welfare workers, police and criminal justice services and other relevant community agencies.

8. Link with other sectors to facilitate the increasingly regarded concept of recovery.

9. Monitor community mental health: relevant indicators of early psychosis

10. Support more research
Initiate school and workplace mental health programmes. Encourage the activities of nongovernmental organisations
Include mental disorders in basic health information systems. Survey high-risk population groups.
Improve awareness and detection for early psychosis in the community.

Strengthen school and workplace Development of effective youth-friendly integrated mental health programmes. Links are developed between substance misuse and psychosis care and treatment programmes. programmes for supporting access to work, education and recreation. Effectiveness should be demonstrated, e.g.

(a) client satisfaction;

(b) employment rates;

(c) educational achievement;

(d) access to accommodation.

Develop integrated programmes for care and treatment of those with a combination of early psychosis and substance misuse problems with a single unified service system.

Develop advanced mental health monitoring systems for early psychosis that can measure duration of untreated psychosis; positive recovery outcomes; morbidity in health and social terms; mortality (including suicides); cost and burden.

Monitor effectiveness and cost-effectiveness of prevention and treatment programmes in early psychosis.

Conduct studies on the prevalence, course, outcome and impact of early psychosis in the community.
Institute effectiveness and cost-effectiveness studies for management of early psychosis. (a) on causes of psychosis;

(b) on prevention of psychosis;

(c) on novel psychosocial and psychopharmacological interventions;

(d) on service delivery models (e.g. urban/rural/ dispersed rural);

(e) about prevalence and needs of children and adolescents with early psychosis (aged under 16) both in terms of early detection and intervention implications. 
represent a major element of respect of individuals' rights to citizenship and social inclusion.

\section{VISION}

Fundamental objectives derived from this statement are to:

(a) Challenge stigmatising and discriminatory attitudes so that young people are not disadvantaged by their experiences and are truly included in their local communities.

(b) Generate optimism and expectations of positive outcomes and recovery so that all young people with psychosis and their families achieve ordinary lives.

(c) Raise wider societal awareness about psychosis and the importance of early intervention.

(d) Attract and encourage practitioners from a wide range of health, social, non-governmental agencies (e.g. charitable, voluntary and youth), educational and employment services to reflect on how they can better contribute to supporting young people with psychosis, their families and their friends.

\section{VALUES}

Programmes for the detection and treatment of early psychosis should value:

(a) Respect of the right to recovery and social inclusion and support for the importance of personal, social, educational and employment outcomes.

J. BERTOLOTE, MD, World Health Organization; P. McGORRY, MD, PhD, FRCP, FRANZCP, International Early Psychosis Assocation

Correspondence: Professor P. McGorry, ORYGEN Research Centre, Locked Bag 10/35, Poplar Road, Parkville, Victoria 3052, Australia. Tel +61 39342 2921 ; fax +61 39342 2921 ; e-mail: mcgorry@ariel.unimelb.edu.au

(b) Respect of the strengths and qualities of young people with a psychosis, their families and communities, encouraging ordinary lives and expectations.

(c) Services that actively partner young people, their families and friends to place them at the centre of care and service delivery, at the same time sensitive to age, phase of illness, gender, sexuality and cultural background.

(d) Use of cost-effective interventions.

(e) Respect of the right for family and friends to participate and feel fully involved.

\section{A 5-YEAR PROGRAMME OF ACTION}

This consensus statement describes (Table 1) a number of 5-year measurable outcomes that an individual and their family can expect from services who have successfully implemented a comprehensive and effective programme.

\section{TECHNICAL INTERVENTIONS}

Attaining these 5-year outcomes will require underpinning by modern technical interventions and actions: (a) A series of interventions has demonstrated efficacy in the management of early psychoses, among which there are pharmacological and psychosocial approaches, the latter addressed to both patients and family members (International Early Psychosis Association - IEPA - consensus statement, 2002).

(b) Some of these interventions are specified, by level of resources in a given area, in Table 2, according to the recommendation put forward by the World Health Organization's World Health Report 2001. In the same Annex are found strategic actions needed/useful for the implementation of those interventions.

\section{REFERENCES}

Consensus Statement on Principles and Practice in Early Psychosis (2002) In Implementing Early Intervention in Psychosis: A Guide to Establishing Early Psychosis Services (eds J. Edwards \& P. D. McGorry), pp. 145-155. London: Dunitz.

The Newcastle Early Psychosis Declaration (2002) http: / /www.rethink.org/newcastledeclaration

World Health Organization (2002) World Health Report 200I. Mental Health: New Understanding. New Hope. Geneva: WHO 